\title{
THE APPLICATION OF STEREOLOGICAL METHODS FOR THE QUANTITATIVE ANALYSIS OF THE ATHEROSCLEROTIC LESIONS IN RABBITS
}

\author{
Petr Nachtigal ${ }^{1}$, Vladimír SEMEckÝ ${ }^{1}$, ANDrea GojovÁ ${ }^{1}$, Martin KopeckÝ ${ }^{1}$, ViKTor \\ BENEŠ ${ }^{2}$ AND RADKA JǓZKOVÁ ${ }^{2}$ \\ ${ }^{1}$ Department of Biological and Medical Sciences, Charles University in Prague, Faculty of Pharmacy in Hradec \\ Králové, Czech Republic, ${ }^{2}$ Department of Probability and Mathematical Statistics, Charles University in \\ Prague, Faculty of Mathematics and Physics, Sokolovská 83, 18675 Praha 8, Czech Republic \\ e-mail: nachti@faf.cuni.cz, semecky@faf.cuni.cz, gojova@faf.cuni.cz,95KOM3505@faf.cuni.cz, \\ benesv@karlin.mff.cuni.cz,rjuz7233@ss1000.ms.mff.cuni.cz \\ (Accepted October 29, 2002)
}

\begin{abstract}
Atherosclerosis is a chronic metabolic and inflammatory disease of vascular arteries. In some cases, it comes the accumulation of the inflammatory cells and extracellular matrix in vessel intima, which lead to the narrowing of the vessel lumen. The aim of this study was introduce stereological methods for the quantification of atherosclerotic changes in the aorta of New Zealand White rabbits in dependence on the period of feeding with atherogenic diet containing $0.4 \%$ of cholesterol. The Cavalieri estimator was used for the estimation of the volume of atherosclerotic lesions. The area of the atherosclerotic lesions and the area fraction of free vessel lumen were determined with the image analysis software Lucia. The principle of the point counting method was used for the estimation of the volume fraction of collagen in atherosclerotic lesions and the principle of the optical disector was used for the estimation of the number of cells per unit volume in atherosclerotic lesions. The results obtained by the stereological methods confirmed the induction and progression of atherogenic changes in rabbit vessel wall in cholesterol fed animals. The methods applied in this study will be used in our prospective studies where the effect of hypolipidemic drug treatment on atherogenic process will be monitored.
\end{abstract}

Keywords: atherosclerosis, Cavalieri principle, collagen, disector, number of cells per unit volume, volume estimation.

\section{INTRODUCTION}

Atherosclerosis represents an arterial disease process affecting large and medium-sized elastic and muscular arteries and involves primarily the intima of the vessels. There are several atherogenic factors that may lead to the initiation and progression of atherosclerosis, including genetic predisposition, hyperlipidemic states (LDL, VLDL) and hemodynamic stress (hypertension). There are several predisposed sites of the localization of atherosclerosis. The coronary arteries, the major branches of aortic arch, the thoracic aorta, the abdominal aorta, and the renal arteries are sites susceptible to the atherosclerotic process (Spiros et al., 1999). Atherogenesis consists of multiple and complex disease processes including endothelial dysfunction that is followed by the infiltration of inflammatory leukocytes, accumulation of cholesterol-laden macrophages, proliferation of smooth muscle cells, and deposition of extracellular matrix in the vessel intima. Accumulation of these inflammatory cells and proliferation of extracellular matrix contributes to the intimal thickening and hence to the narrowing of the vessel lumen and subsequently to the formation of advanced atherosclerotic lesion. This narrowing of the vessel lumen and changes in the structure and the function of the vessels may lead to many clinical complications such as ischemia of the heart, brain or extremities and together with thrombosis or plaque rupture may result in the myocardial infarction (Ross, 1999).

The cholesterol-fed rabbit is a widely used model for the experimental atherosclerosis research. Russian scientist Nikolaj Nikolajewitsch Anitschkow firstly used New Zealand White rabbits as a model of atherosclerosis at the beginning of the century (Finking and Hanke, 1997). In normal rabbits, cholesterol feeding leads to the induction of hypercholesterolemia and subsequently to alterations in the vessel wall intima and formation of atherosclerotic lesion. 
The quantification of the atherosclerotic lesion is used for the objective characterization of atherogenic process. The volume of the atherosclerotic lesion, the intima media thickness are geometric parameters that describe the narrowing of the vessel lumen (Wendelhag et al., 1996). Mechanical strength (stability) of atherosclerotic plaques is primarily determined by the collagen content and distribution (Wight, 1996). On the other side high cellular density in the lesion, especially increased amount of the macrophages is accompanied by the synthesis of proteolytic enzymes that destroy collagen and thereby contribute to the weakening of the plaque (Rekhter et al., 2000). The quantification of the collagen content in the atherosclerotic lesion might be important marker that characterizes the stability of the atherosclerotic plaque (Richard, 2000). The quantification of the cellular density in the atherosclerotic arteries by using image analysis or disector principle might be useful for studying apoptosis and proliferation of the cells (Schneider et al., 2000; Rizzoni et al., 1998).

In the present study, we focused on the application of computer-assisted image analysis and stereological principles for the quantification of atherosclerotic changes in vessel wall intima. Estimations of the volume of atherosclerotic lesions, the area fraction of free vessel lumen (AFFVL), the collagen content, and the cellular density in atherosclerotic lesions were carried out.

\section{MATERIALS AND METHODS}

Twenty-four adult male New Zealand White rabbits ( 2.0 to $3.0 \mathrm{~kg}$, BIOTEST Konárovice) were randomly assigned into three groups. In each group, six rabbits consumed an atherogenic diet $(0.4 \%$ cholesterol, $3 \%$ fat, $19 \%$ proteins) and two rabbits consumed standard diet (control animals). Rabbits obtained 150 grams of atherogenic or standard diet per day respectively and food intake was monitored every morning. The first group of animals was fed for 1 month, the second group was fed for 2 months, and the third group was fed for 3 months. On the day of the experiment, rabbits were anesthetized with intramuscular dose of ketamine (1 $\mathrm{ml} / 1 \mathrm{~kg}$ ) and euthanasied with overdose of sodium pentobarbital $(500 \mathrm{mg}$ ad $10 \mathrm{ml}$ of distilled water) administered into the auricular vein. The histological, immunohistochemical and stereological analysis was performed in $3 \mathrm{~cm}$ long segments of aortic arch, thoracic aorta, and abdominal aorta. Arteries were fixed with Baker's fixative and embedded in paraffin.

Morphometric analysis and photodocumentation were provided by means of computer-assisted image analysis system with LUCIA software (Laboratory Imaging Prague s.r.o.) and with the stereological software ELLIPSE and DISECTOR (ViDiTo, Zoltan Tomori, Kosice, Slovak Republic, tomori@saske.sk).

Cavalieri principle was used for the estimation of the volume $\mathrm{V}$ of the atherosclerotic lesions in a reference volume. A total number $1=72$ of consecutive serial cross sections were cut into $5 \mu \mathrm{m}$ thick slices (the thickness of the slices were set on Leica DSC 1 disc automatic microtome) which gives us $0.36 \mathrm{~mm}$ long piece of the vessel called the reference volume. A systematic uniform random sampling in the reference volume was used. The first section was randomly positioned and then each ninth section was used, thus $\mathrm{m}=8$ sections were used for the estimation. All these sections were stained with orcein and Weigert hematoxylin for the estimation of the lesion volume in the reference volume. The Cavalieri estimator is then simply $\mathrm{T}$ times the total section area, see Eq. 1 (Roberts et al., 1993)

$$
\text { est } V=T *\left(\mathrm{~A}_{1}+\mathrm{A}_{2}+\ldots+\mathrm{A}_{\mathrm{m}}\right) \mathrm{mm}^{3},
$$

where $\mathrm{T}=0.045 \mathrm{~mm}$ is the distance between two measured sections, and $A_{i}$ is the area of the atherosclerotic lesion in $\mathrm{i}$-th measured section. The sectioned areas of the lesions were not measured by point counting method as previously (Semecký et al., 1998), but were measured by Image analysis software LUCIA.

The choice of the number of sections is based on the analysis of the coefficient of error (CE) of the volume estimator. The empirical resampling method in the reference volume was used to determine the coefficient of error $\mathrm{CE}$ (estV) of the Cavalieri estimator with respect to number of sections according to the Eq. 2 and Eq. 3 (McNulty et al., 2000).

$$
\mathrm{CE}^{2}=\frac{1}{\mathrm{~T}^{*} \mathrm{~V}^{2}} \sum\left(\mathrm{V}_{\mathrm{i}}\right)^{2}-1, \quad \mathrm{i}=1 \ldots \mathrm{k}
$$

where $k=\frac{l}{m}$ and i denotes the starting position of a slice in the resampling. Here

$$
V_{i}=T * \sum_{j=0}^{m-1} A_{j k+i}
$$

The area fraction of free vessel lumen (AFFVL) is a parameter that characterizes the narrowing of the vessel lumen at a section and it was evaluated according to Eq. 4.

$$
A F F V L=\left(1-\frac{\mathrm{A}(\text { lesion })}{\mathrm{A}(\text { lumen })}\right) * 100 \%,
$$

where $A$ (lesion) is the area of atherosclerotic lesion and $A$ (lumen) is the area of the total vessel lumen (including lesion), see Fig. 1. 


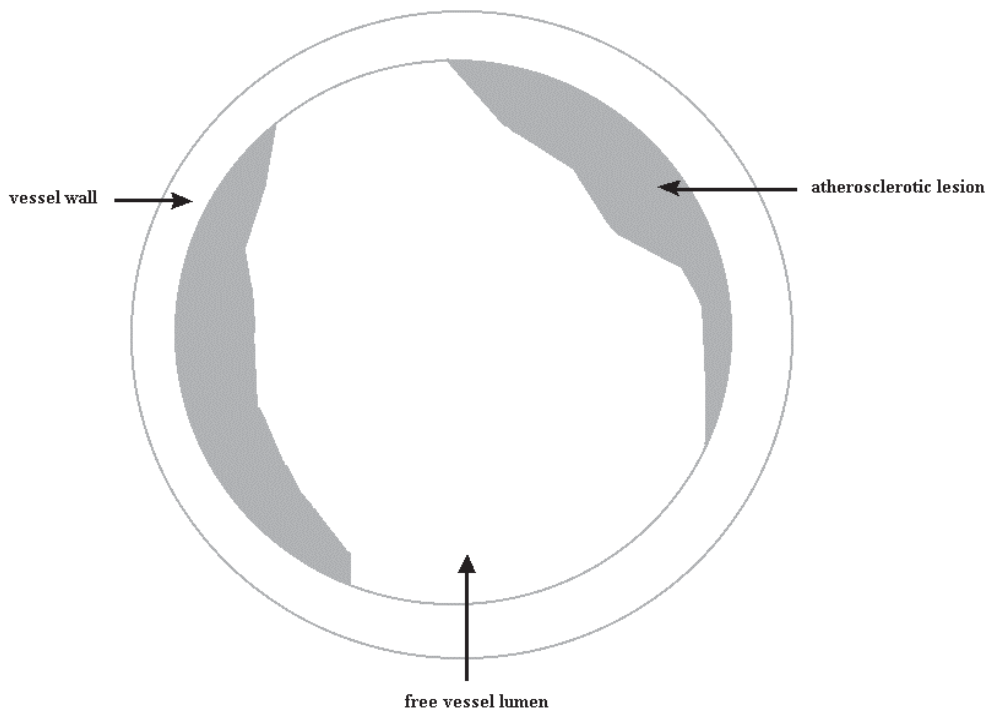

Fig. 1. The scheme of transverse vessel section. Free vessel lumen is characterized by the AFFVL parameter.

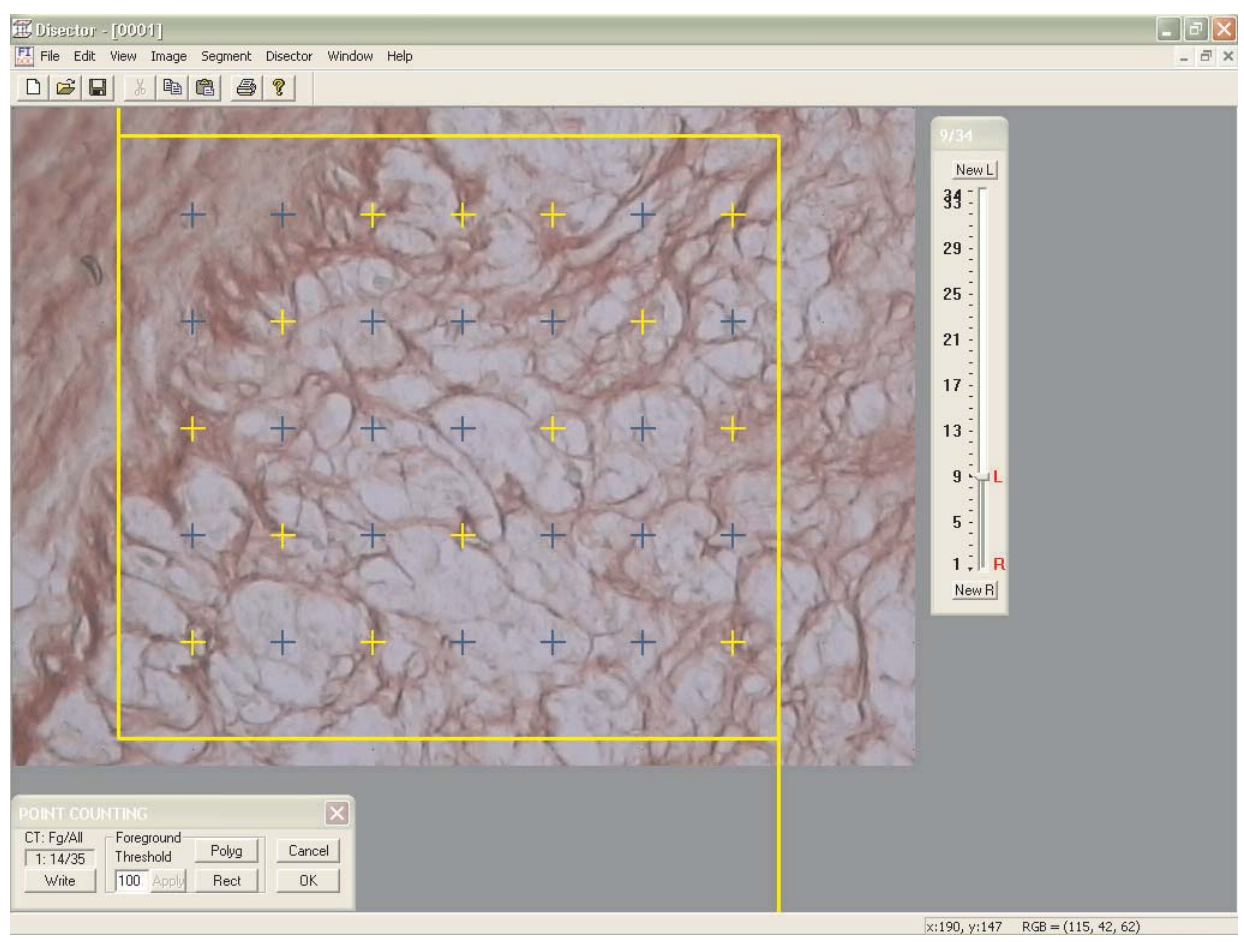

Fig. 2. The principle of the quantification of the volume fraction of collagen in the atherosclerotic lesions. The test points hitting the collagen bundles in the atherosclerotic lesion (yellow points).

Six slices were stained with Sirius red for the vizualization of collagen. The Sirius red is a histological dye suitable for the staining of total collagen in arterial wall. The systematic uniform random sampling and the principle of the point-counting method (Weibel, 1979), were used for the estimation of the volume fraction of total collagen in the atherosclerotic lesions. The principle of the collagen quantification is shown in Fig. 2. At first, the number of test points hitting the area of the lesions was counted. Secondly, the number of test points hitting the collagen bundles (red staining) inside the atherosclerotic lesions was counted. More than 200 test points hitting the collagen bundles in the lesions were counted per vessel (Gundersen et al., 1997). The volume fraction of the collagen in the atherosclerotic lesions is then, 


$$
\text { est } V_{V}=\frac{A(\text { collagen })}{A(\text { lesion })} * 100 \% \text {, }
$$

where $A$ (collagen) is the area of the collagen bundles in the lesion and $A$ (lesion) is the area of the atherosclerotic lesion in the section.

The number of cells per unit volume in the atherosclerotic lesion was estimated by using the stereological principle of the optical disector (Gundersen, 1986). Twenty $\mu \mathrm{m}$ thick (the thickness of slices was set on Leica DSC 1 disc automatic microtome) consecutive cross sections were cut and stained with Hill hematoxylin for the visualization of the cell nuclei. The thickness of the specimen was estimated as a mean thickness in 5 locations, uniformly distributed within the specimen, measured under a Bio-Rad MRC 600 laser scanning confocal microscope, equipped with an automatic $z$-focus stepping motor. Series of optical sections were made by focusing through thicker physical sections in an optical microscope and counted by DISECTOR program (Tomori et al., 2001). 48 disectors and more than 100 cells per vessel were counted. The number of cells per unit volume was then estimated according to Eq. 6

$$
\operatorname{estN}_{V}=\frac{\sum_{i=1}^{n} Q_{i}}{\sum_{i=1}^{n} P i} * \frac{p}{a^{\prime} * h},
$$

where $\mathrm{n}$ is the number of disectors, $\mathrm{Q} i(i=1, \ldots, \mathrm{n})$ is the number of cells in the atherosclerotic lesion sampled by the $i$-th disector, $\mathrm{P} i(\mathrm{i}=1, \ldots, \mathrm{n})$ is the number of points of the $p$-point grid in $i$-th the frame hitting the lesion section, $\mathrm{a}^{\prime}\left(\mathrm{mm}^{2}\right)$ is the area of the frame, and $\mathrm{h}(\mathrm{mm})$ is the distance between disector planes (disector height).

Finally, statistical tests were used for the comparison of stereological parameters estimated from three groups of animals. The experiment yields three independent samples for each parameter. Since the sample $\mathrm{n}=6$ is small and normality assumption is strongly violated a Kruskal-Wallis statistical nonparametric test was used to test the null hypothesis that all means are equal. When rejected this analysis followed by pairwise comparison procedures by Nemenyi Method (Anděl, 1978). Either a p-value is evaluated or a classical test with given significance level $\alpha$ is used.

\section{RESULTS}

The empirical resampling method for the determination of coefficient of error of the Cavalieri estimator with respect to number of sections showed that eight sections are sufficient for the estimation of the volume of the atherosclerotic lesions in $0.36 \mathrm{~mm}$ long piece of the vessel, see Fig. 3.

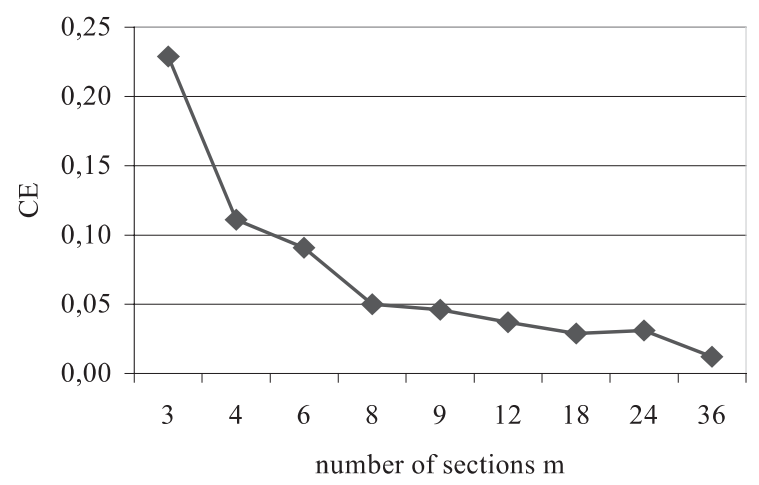

Fig. 3. The coefficient of error of the Cavalieri estimator with respect to the number of sections estimated from abdominal aorta of rabbit fed by atherogenic diet for three months. Here CE(est V) $=0.05$ for eight sections.

As we can see in Fig. 4, the volumes of the atherosclerotic lesions in measured segments of aortic arch, thoracic aorta and abdominal aorta were increasing depending on the period of feeding with cholesterol diet. The mean volumes of the lesions from aortic arch were $0.0336 \mathrm{~mm}^{3}, 0.1199 \mathrm{~mm}^{3}$, $0.7802 \mathrm{~mm}^{3}$, from thoracic aorta $0.0017 \mathrm{~mm}^{3}, 0.0418$ $\mathrm{mm}^{3} 0.1400 \mathrm{~mm}^{3}$ in rabbits fed by atherogenic diet for one, two and three months, respectively. There were no changes in the intima of the vessels in abdominal aorta of rabbits fed by diet for one month. The mean volumes of the lesions in abdominal aorta were $0.0139 \mathrm{~mm}^{3}$ and $0.0415 \mathrm{~mm}^{3}$ in rabbits fed by atherogenic diet for two and three months, respectively. There were no changes in the intima of the vessels in control group. There were statistically significant differences in the mean volumes of atherosclerotic lesions between rabbits fed by atherogenic diet for three months and two months, three months and one month in aortic arch and for three months and one month in thoracic and abdominal aorta, respectively, see Table 1 . 


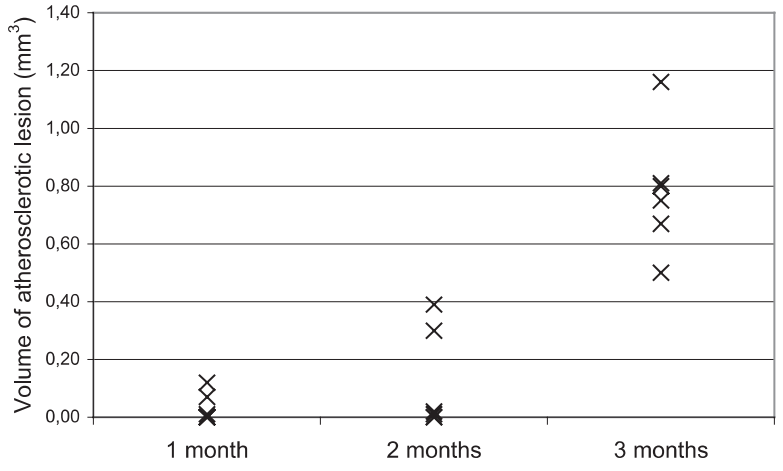

(A) Aortic arch: Three rabbits in one-month diet and two rabbits in two months diet had no lesions.

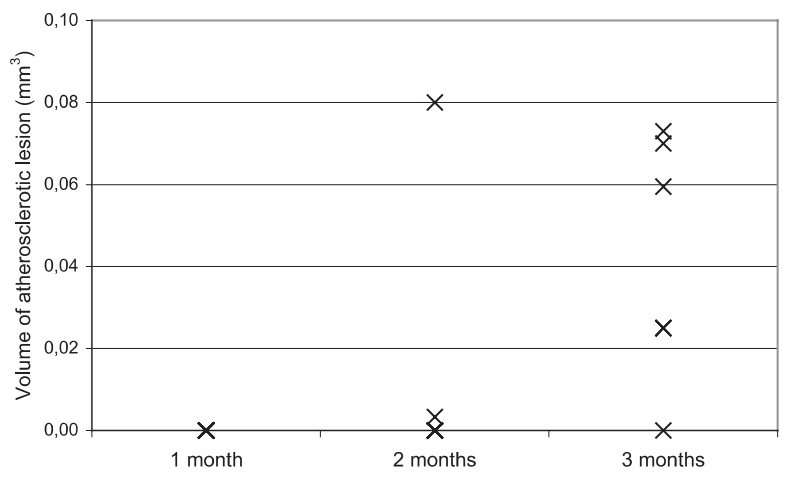

(B) Thoracic aorta: Five rabbits in one-month diet, three rabbits in two months diet and one rabbit in three months diet respectively, had no lesions.

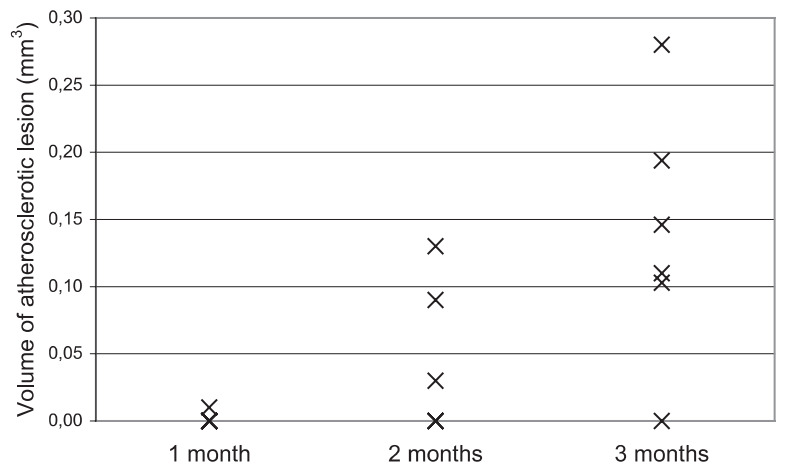

(C) Abdominal aorta: No lesions were found in one-month diet. Four rabbits in two months diet and one rabbit in three months diet had no lesions. Two rabbits in three months diet had the same volume of the lesion, namely $0.03 \mathrm{~mm}^{3}$.

Fig. 4. The volumes of the atherosclerotic lesions from 18 rabbits, 6 in each group (the period of feeding) in aortic arch (A), thoracic aorta (B) and abdominal aorta $(C)$.
The mean AFFVL was decreased depending on the period of feeding with atherogenic diet in all measured segments of rabbit aorta, which is comprehensible due to the increasing area of the lesions in the vessel lumen, see Fig. 5. Rabbits treated by atherogenic diet for one month showed only negligible thickening of intima in vessels and therefore the mean AFFVL was very high. The mean AFFVL was $98.5 \%$ in aortic arch and $99.9 \%$ in thoracic aorta. The mean AFFVL in abdominal aorta was $100 \%$, no lesions were found. Rabbits treated by atherogenic diet for two months showed an increasing thickening of the intima and therefore the mean AFFVL was slightly decreased, namely $95.4 \%$ in aortic arch, $97.8 \%$ in thoracic aorta and $97.5 \%$ in abdominal aorta. There was accentuated increase of the areas of lesions in rabbits fed by atherogenic diet for three months and of course the mean AFFVL was markedly decreased, namely $71.2 \%$ in aortic arch, $89.6 \%$ in thoracic aorta and $87.8 \%$ in abdominal aorta. The mean AFFVL in control group was $100 \%$ because no atherosclerotic lesions were found. There were statistically significant differences between rabbits fed on diet for three months and one month in all measured segments of rabbit aorta. In addition, there was statistical significant difference in aortic arch between rabbits fed on atherogenic diet for two and three months, see Table 1.

Table 1. Results of the Kruskal-Wallis nonparametric test. There is a statisticaly significant difference if $Q \geq 5.99$ on the level $\alpha=0.05$. This classical test does not enable to reject the null-hypothesis on the third row, however the $p$-value $=0.054$ is small.

\begin{tabular}{lrc}
\hline & $\mathrm{Q}$ & $\mathrm{p}$ \\
\hline Lesion volume - aortic arch & 10.05 & 0.007 \\
Lesion volume - thoracic aorta & 6.72 & 0.035 \\
Lesion volume - abdominal aorta & 5.84 & 0.054 \\
AFFVL - aortic arch & 11.49 & 0.003 \\
AFFVL - thoracic aorta & 7.46 & 0.024 \\
AFFVL - abdominal aorta & 6.89 & 0.032 \\
\hline
\end{tabular}




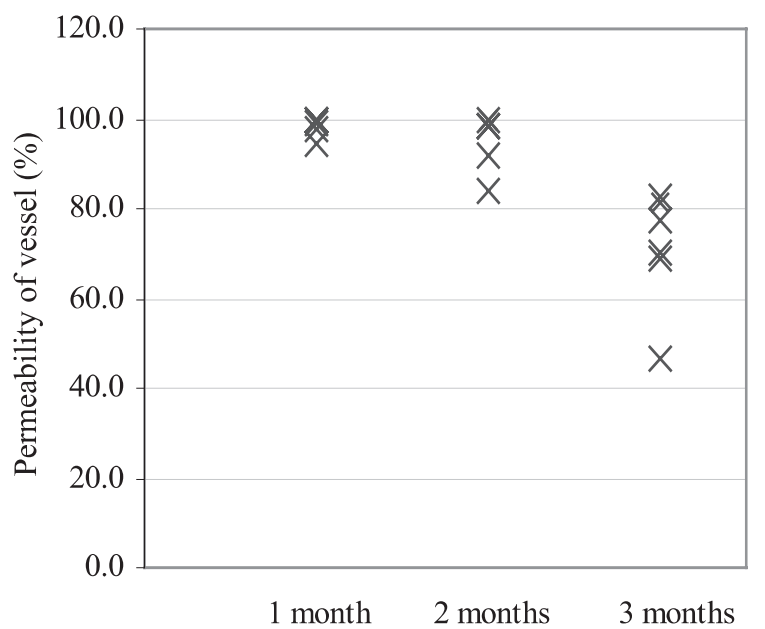

(A) Aortic arch: Three rabbits in one-month diet and two rabbits in two months diet had no lesions, thus AFFVL was $100 \%$. Two rabbits in two months diet had almost the same AFFVL, namely $98.5 \%$ and $98.3 \%$.

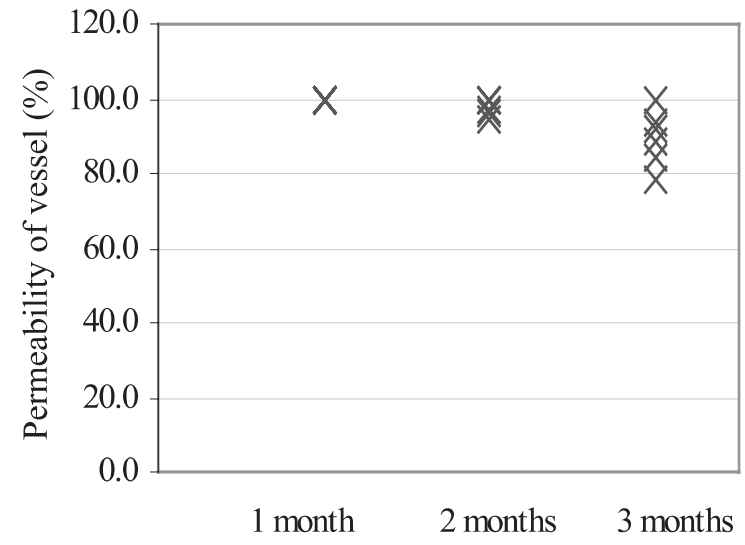

(B) Thoracic aorta: Five rabbits in one-month diet, three rabbits in two months diet and 1 rabbit in three months diet respectively, had no lesions.

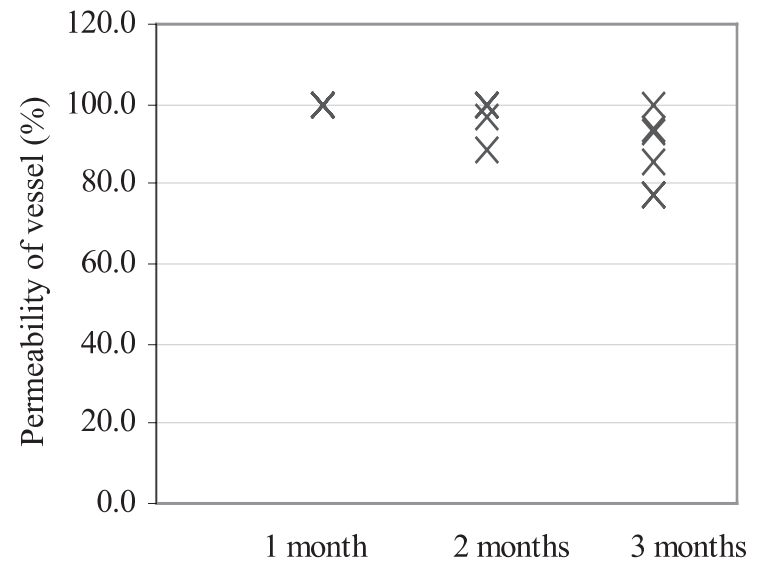

(C) Abdominal aorta: No lesions were found in one-month diet. Four rabbits in two months diet and one rabbit in three months diet had no lesions. Two rabbits in three months diet had almost the same AFFVL, namely $77.1 \%$ and $77.0 \%$.

Fig. 5. The values of the AFFVL from 18 rabbits, 6 in each group (the period of feeding) in aortic arch (A), thoracic aorta (B) and abdominal aorta (C).
Fig. 6 shows particular examples of the vessel sections from all groups of rabbits. Note that the control group of rabbits has no visible changes in the vessel intima. Small infiltration in the intima was visible after one month of feeding but these lesions do not affect the AFFVL very much. Rabbits treated with atherogenic diet for two and three months respectively, showed more accentuated changes in the intima. All these figures correspond to the results described above.

The quantification of the volume fraction of collagen in the atherosclerotic lesions showed only slight differences among experimental groups and among different parts of the aorta, respectively. However, it should be stated that the actual values of volume fractions of collagen are over-estimates (Holmes effect). As seen in the Table 2 there is a very small growth of the volume fraction of collagen in measured atherosclerotic vessels with the period of feeding. No estimation of the volume fraction of collagen was made in control group (no lesions were found) and in vessels with AFFVL higher than 95\%, respectively. Therefore, no overall statistical analysis was performed due to the small numerus.

Table 2. The volume fraction of collagen in the atherosclerotic lesions from all experimental rabbits, each parts of aorta.

\begin{tabular}{ccc}
\hline \multicolumn{3}{c}{ Aortic arch (\%) } \\
\hline 1 month & 2 months & 3 months \\
\hline 44.5 & 44.4 & 55.8 \\
- & 45.2 & 46.0 \\
- & - & 50.6 \\
- & - & 52.9 \\
- & - & 49.0 \\
- & - & 58.3 \\
\hline \multicolumn{3}{c}{ Thoracic aorta (\%) } \\
\hline 1 month & 2 months & 3 months \\
\hline- & 43.2 & 52.1 \\
- & - & 48.3 \\
- & - & 42.9 \\
- & - & 46.8 \\
- & - & 52.2 \\
- & - & - \\
\hline \multicolumn{4}{c}{ Abdominal aorta $(\%)$} \\
\hline- & 2 months & 3 months \\
\hline- & 50.6 & 43.4 \\
- & - & 56.5 \\
- & - & 50.2 \\
- & - & 50.8 \\
- & - & 59.0 \\
\hline
\end{tabular}




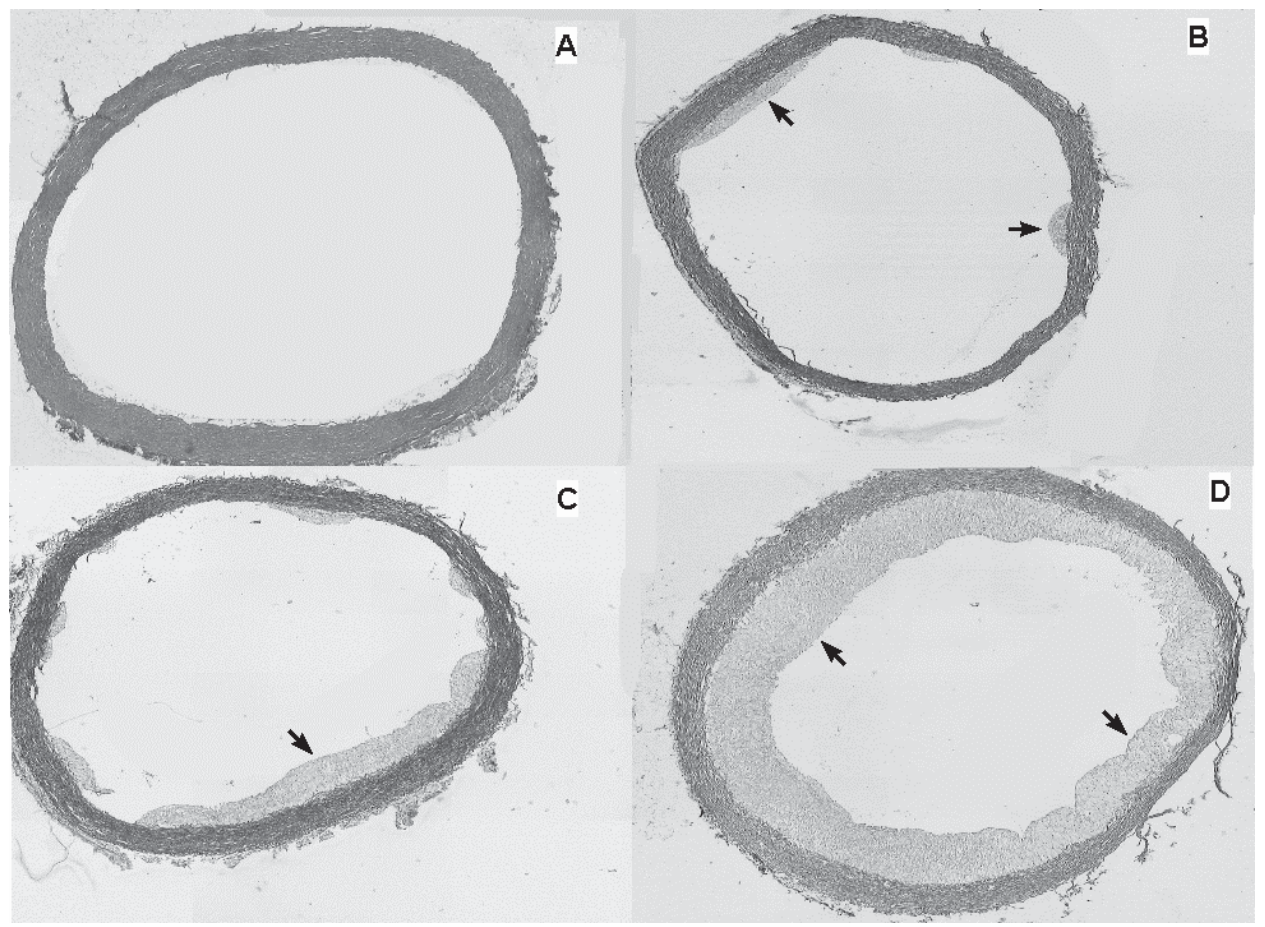

Fig. 6. Photomicrographs of the aortic wall. There is no lesion in the vessel lumen of control group (A). There is only a small infiltration in the vessel intima in rabbits fed on atherogenic diet for 1 month (B) (arrowheads). The areas of the lesions are increased in rabbits fed on atherogenic diet for 2 months $(C)$ and 3 months $(D)$, respectively (arrowheads). Original magnification $\times 40$.

The number of cells was estimated per unit volume, i.e., $1 \mathrm{~mm}^{3}$. The results of the mean number of cells per unit volume are shown in the Table 3. There are only small differences among experimental groups in all parts of rabbit aorta but it seems that the number of cells per unit volume was slightly increased in abdominal aorta. No estimation of the number of cells per unit volume was made in control group (no lesions were found) and in vessels with AFFVL higher than $95 \%$, respectively. Therefore, no statistical analysis was performed due to the small numerus.

Moreover, it was not our purpose to determine differences among the control and the experimental groups, but just to introduce and test proper methodology that we could successfully apply in our further studies.
Table 3. The number of cells per unit volume (in $1 \mathrm{~mm}^{3}$ ) in the atherosclerotic lesions from all experimental rabbits, each parts of aorta.

\begin{tabular}{ccc}
\hline \multicolumn{3}{c}{ Aortic arch (\%) } \\
\hline 1 month & 2 months & 3 months \\
\hline 305461 & 305859 & 176185 \\
- & 176634 & 244311 \\
- & - & 230884 \\
- & - & 248281 \\
- & - & 298329 \\
- & - & 217769 \\
\hline \multicolumn{3}{c}{ Thoracic aorta (\%) } \\
\hline 1 month & 2 months & 3 months \\
\hline- & 202296 & 294805 \\
- & - & 199346 \\
- & - & 279028 \\
- & - & 251717 \\
- & - & 252585 \\
- & - & - \\
\hline & Abdominal aorta (\%) \\
\hline month & 2 months & 3 months \\
\hline- & - & 263301 \\
- & - & 314972 \\
- & - & 233178 \\
- & - & 241753 \\
- & - & - \\
\hline
\end{tabular}




\section{DISCUSSION}

The aim of this study was to apply computerassisted image analysis and stereological methods for the quantification of the atherosclerotic changes in rabbit vessel wall. Morphometric studies of atherosclerotic changes in vessels are widely used for the description of the progression of atherosclerosis in rabbits (Schwenke and Carew, 1989) and humans (Kopalov et al., 1996; Goubergrits et al., 2001). Atherosclerotic plaque area, intima media thickness or arterial wall thickness are frequent parameters used in the quantification of atherosclerosis (Wendelhag et al., 1992; Fernandez-Britto et al., 1999). The wide spectrum of methods is used including noninvasive ultrasound methods (Wendelhag et al., 1993), magnetic resonance imaging (Underwood et al., 1993), and computer-assisted image analysis (Bonithon-Kopp et al., 1996; Aly and Bishop, 2000; Goubergrits et al., 2002).

The estimations of the volume of the atherosclerotic lesion and the AFFVL were used for the quantification of atherosclerotic changes in vessels in our study. The combination of the Cavalieri principle and Image analysing software LUCIA was applied. The results of the estimations of the volume of atherosclerotic lesions and the AFFVL showed correlation between progression of atherosclerotic changes in each parts of aorta and the period of feeding with atherogenic diet. It seems that atherogenic changes were more accentuated in aortic arch than in thoracic or abdominal aorta, respectively.

The quantification of the collagen in the atherosclerotic lesion is widely used in the atherosclerotic research especially in studies focusing on the stability and behaviour of the atherosclerotic plaque (Abela and Picon, 1995; Ruengsakulrach et al., 1999). Several methods were developed including computer-assisted image analysis (Lafont et al., 1999), confocal fluorescence microscopy (Tatjes et al., 2000), video-morphometric system (Plenz et al., 1999) or video-microdensitometry (Kratky et al., 1999). We used the stereological software ELLIPSE, systematic uniform random sampling, and the principle of point-counting method for this quantification. This method is very fast and comfortable and minimizes problems with negative background staining that usually interferes with positive one if we use computer-assisted image analysis.

The total number of cells per unit volume in the atherosclerotic lesion can be quantified by using image analysis software together with fluorescence and confocal microscopy (Taatjes et al., 2000). Most of these methods are based on the principle of counting cell profiles in 2D tissue sections, which is not correct because the results depend on the height of the cells and the sectioning plane (larger cells are more likely to be hit by the sectioning plane than smaller ones). In our experiment, the number of cells per unit volume was estimated by using the principle of optical disector, which enables us to count particles irrespective of their size and shape (Sterio, 1984).

The quantification of the volume fraction of collagen and the number of cells per unit volume was performed only in fibromuscular atherosclerotic lesions that fill in more than $5 \%$ of the vessel lumen (the AFFVL $\leq 95 \%$ ). The results of the estimations of the volume fraction of collagen and the number of cells per unit volume showed only negligible differences among experimental groups and particular parts of aorta, respectively. These outcomes are comprehensible because all measured lesions were of fibromuscular type. The most important for us is the fact that we will be able to apply these stereological principles for the estimation of the volume fraction of collagen and the number of cells per unit volume in atherosclerotic lesion in our prospective studies focusing on stability and nonstability of atherosclerotic plaque.

In conclusion, the aim of this study was to introduce stereological methods and computer-assisted image analysis for the estimation of some parameters that characterise the atherogenic process, including the volume of the atherosclerotic lesions, the AFFVL, the volume fraction of collagen and the number of cells per unit volume. All these methods have proved to be very useful in our prospective studies where the effect of hypolipidemic drugs on the atherogenic process will be studied.

\section{ACKNOWLEDGEMENTS}

The authors wish to thank Mrs. B. Šlechtová, Mrs. Ing. Z. Müllerová for her skilful technical assistance and Mrs. Dr. L. Kubínová for her splendid assistance regarding stereological methods. This work was supported by GAUK grant No. 92/200C, MŠMT grants 111600002 and 113200008 .

\section{REFERENCES}

Abela GS, Picon PD (1995). Triggering of plaque disruption and arterial thrombosis in an atherosclerotic rabbit model circulation. 91:776-84.

Aikawa M, Rabkin R, Okada Y, Voglic SM (1998). Lipid lowering by diet reduces matrix metalloproteinase activity and increases collagen content of rabbit 
atheroma. A potential mechanism of lesion stabilization. Circulation 97:2433-44.

Aly S, Bishop CHC (2000). An objective characterization of atherosclerotic lesion: an alternative method to identify unstable plaque. Stroke 31:1921-4.

Anděl J (1978). Matematická statistika. SNTL/ALFA, Praha.

Bonithon-Kopp C, Touboul PJ, Berr C, Leroux C, Mainard F, Courbon D, Ducimetiere P (1996). Relation of intima-media thickness to atherosclerotic plaques in carotid arteries. The Vascular Aging (EVA) Study. Arterioscler Thromb Vasc Biol 16(2):310-6.

Fernandez-Britto JE, Wong R, Contreras D, Nordet P, Sternby NH (1999). Pathomorphometrical characteristics of atherosclerosis in youth. A multinational investigation of WHO/World Heart Federation (1986-1996), using atherometric system. Nutr Metab Cardiovasc Dis 9(5):210-9.

Finking G, Hanke H (1997). Nikolaj Nikolajewitsch Anitschkow (1885-1964) established the cholesterolfed rabbit as a model for atherosclerosis research. Atherosclerosis 135:1-7.

Goubergrits L, Affeld K, Fernandez-Britto J, Falcon L (2002). Atherosclerosis and flow in carotid arteries with authentic geometries. Biorheology 39(3-4):519-24.

Goubergrits L, Affeld K, Fernandez-Britto J, Falcon L (2001). Atherosclerosis in the human common carotid artery. A morphometric study of 31 specimens. Pathol Res Pract 197(12):803-9.

Gundersen HJG (1986). Stereology of arbitrary particles. A review of unbiased number and size estimators and the presentation of some new ones, in memory of William R. Thompson. J Microsc 143:3-45.

Gundersen HJG, Jensen EB (1997). The efficiency of systematic sampling in stereology and its prediction. $\mathrm{J}$ Microsc 147:229-63.

Kopalkov V, Polishchuck R, Bannykh S, Solovjev P (1996). Atherosclerosis-prone branch regions in human aorta: microarchitecture and cell composition of intima. Arteriosclerosis 122:173-89.

Kratky RG, Ivey J, Roach MR (1999). Local changes in collagen content in rabbit aortic atherosclerotic lesions with time. Atherosclerosis 143:7-14.

Lafont A, Durand E, Samuel JL, Besse B (1999). Endothelial dysfunction and collagen accumulation two independent factors for restenosis and constrictive remodeling after experimental angioplasty. Circulation 100:1109-15.

McNulty V, Cruz-Orive LM, Roberts N, Holmes CJ, GualArnau X (2000). Estimation of brain compartment volume from MR Cavalieri slices. J Comput Assist Tomogr 24(3):466-77.

Plenz G, Dorszewski A, Breithardt G, Robenek H (1999). Expression of type VIII collagen after cholesterol diet and injury in the rabbit model of atherosclerosis. Arterioscler Thromb Vasc Biol 19:1201-9.
Rekhter MD, Hicks GW, Brammer DW, Hallak H, Kindt E, Chen J (2000). Hypercholesterolemia causes mechanical weakening of rabbit atheroma: local collagen loss as a prerequisite of plaque rupture. Circ Res 86:101-8.

Richard TL (2000). Plaque stabilization: the role of lipid lowering. Inter J of Cardiol 74:S11-S15.

Rizzoni D, Porteri E, Piccoli A, Castellano M, Bettoni G, Muiesan M (1998). Effects of Losartan and Enalapril on Small Artery Structure in Hypertensive Rats. Hypertension 32:305-10.

Roberts N, Cruz-Orive LM, Reid NM, Brodie DA, Bourne M, Edwards RTH (1993). Unbiased estimation of human body composition by the Cavalieri method using magnetic resonance imaging. $\mathrm{J}$ Microsc 171(3):239-53.

Ross R (1999). Atheroslerosis - an inflammatory disease. The New England J of Medicine 340:115-26.

Ruengsakulrach P, Sinclair R Komeda M, Raman J, Gordon I (1999). Comparative histopathology of radial artery versus internal thoracic artery and risk factors for development of intimal hyperplasia and atherosclerosis. Circulation 100:139-44.

Schneider DB, Vassalli G, Wen S, Driscoll RM, Andre B (2000). Expression of Fas ligand in arteries of hypercholesterolemic rabbits accelerate atherosclerotic lesion formation. Arterioscler Thromb Vasc Biol 20:298-08.

Schwenke DC, Carew TE (1989). Initiation of atherosclerotic lesions in cholesterol-fed rabbits. II. Selective retention of LDL vs. selective increases in LDL permeability in susceptible sites of arteries. Arteriosclerosis 9:908-18.

Semecký V, Gojová A, Nachtigal P, Langrová K, Beneš V (1998). Stereological analysis of pathological changes in the blood - vessel wall after hypercholesterolemia. Acta Stereol 17:383-8.

Spiros GF, Vivian G, Bauer S (1999). Localization of atherosclerosis: role of hemodynamics. Arch Surg 134:1142-9.

Sterio DC (1984). The unbiased estimation of number and sizes of arbitrary particles using the disector. J Microsc 134:1694-6.

Taatjes DJ, Wadsworth MP, Schneider DJ, Sobel BE (2000). Improved quantitative characterization of atherosclerotic plaque composition with immunohistochemistry, confocal fluorescence microscopy, and computer-assisted image analysis. Histochem Cell Biol 113:161-73.

Tomori Z, Krekule I, Kubínová L (2001). Disector program for unbiased estimation of particle number, numerical density, and mean volume. Image Anal Stereol 20:119-30.

Underwood RS, Underwood RS, Mohiaddin RH (1993). Magnetic resonance imaging of atherosclerotic vascular disease. Am J Hypertens 11:335-9. 
Weibel ER (1979). Stereological methods, Vol 1. Practical methods for biology morphometry. Academic Press.

Wendelhag I, Wiklund O, Wikstrand J (1992). Arterial wall thickness in familial hypercholesterolemia. Ultrasound measurement of intima-media thickness in the common carotid artery. Arterioscler Thromb 12(1):70-7.

Wendelhag I, Wiklund O, Wikstrand J (1993). Atherosclerotic changes in the femoral and carotid arteries in familial hypercholesterolemia: ultrasonographic assessment of intima-media thickness and plaque occurrence. Arterioscler Thromb 13:1404-11.
Wendelhag I, Wiklund O, Wikstrand J (1996). On quantifying plaque size and intima-media thickness in carotid and femoral arteries. Comments on results from a prospective ultrasound study in patients with familial hypercholesterolemia. Arterioscler Thromb Vasc Biol 16(7):843-50.

Wight TN (1996). The vascular extracellular matrix. In: Fuster V, Ross R, Topol EJ, eds. Atherosclerosis and Coronary Artery Disease. Philadelphia, Pa: LippincottRaven Publishers, 421-40. 